\title{
Estimating parametric loss aversion with prospect theory: recognising and dealing with size dependence
}

Article

Accepted Version

Creative Commons: Attribution-Noncommercial-No Derivative Works 4.0

Balcombe, K., Bardsley, N., Dadzie, S. and Fraser, I. (2019) Estimating parametric loss aversion with prospect theory: recognising and dealing with size dependence. Journal of Economic Behavior \& Organization, 162. pp. 106-119. ISSN 0167-2681 doi: https://doi.org/10.1016/j.jebo.2019.04.017 Available at https://centaur.reading.ac.uk/83342/

It is advisable to refer to the publisher's version if you intend to cite from the work. See Guidance on citing.

To link to this article DOI: http://dx.doi.org/10.1016/j.jebo.2019.04.017

Publisher: Elsevier

All outputs in CentAUR are protected by Intellectual Property Rights law, including copyright law. Copyright and IPR is retained by the creators or other copyright holders. Terms and conditions for use of this material are defined in the End User Agreement.

www.reading.ac.uk/centaur 
Central Archive at the University of Reading

Reading's research outputs online 


\section{Estimating Parametric Loss Aversion with Prospect Theory: Recognising and Dealing with Size Dependence.}

Kelvin Balcombe, School of Agriculture, Policy and Development, University of Reading, k.g.balcombe@reading.ac.uk

Nick Bardsley, School of Agriculture, Policy and Development, University of Reading, n.o.bardsley@reading.ac.uk

Sam Dadzie, University of Cape Coast, Ghana, sdadzie@ucc.edu.gh Iain Fraser*, School of Economics, University of Kent, i.m.fraser@kent.ac.uk

\section{*Address for correspondence:}

Iain Fraser, School of Economics, Keynes College, University of Kent, Canterbury, Kent, CT2 7NP, i.m.fraser@kent.ac.uk

\section{Acknowldegements}

The authors thank John Pierson, an Associate Editor and two reviewers for constructive and valuable comments on an ealier version of this manuscript. We also thank seminar participants at the Eastern Arc Workshop, University of Kent, FUR 2016 Warwick, Victoria University, Melbourne and the Melbourne Institute, University of Melbourne. Dadzie was a $\mathrm{PhD}$ when part of the research was conducted at the University of Reading. He was supported by a UK government Commonwealth Scholarship. We also received financial support the University of Cape Coast for conducting the field experiment. 


\title{
Estimating Parametric Loss Aversion with Prospect Theory: Recognising and Dealing with Size Dependence.
}

\begin{abstract}
Parameteric identification of loss aversion requires either the imposition of rotational symmetry on the utility function or a point dependent normalization condition. In this paper, we propose a new approach in which point dependence is reduced by integration over normalization points. To illustrate our approach, we consider a sample of Ghanaian farmers' risk preferences over the gain, loss and mixed domains. Using Bayesian econometric methods, we find support for Prospect Theory albeit with substantial behavioral variation across individuals plus mild overweighting of losses compared to gains. We also show that the majority of respondents are mildly loss averse especially as the size of the payoffs increase.
\end{abstract}

Key Words: Prospect Theory; Loss Aversion; Hierachical Bayes Methods.

JEL: C93, D81, Q16.

\section{Introduction}

In this paper, we propose an approach to estimating loss aversion that recognises that loss aversion cannot be divorced by the scale/size of the gains and losses being compared. We make explicit that, in general, loss aversion cannot be decoupled from the scale of losses versus gains without imposing restrictions that may not be supported by data. A potential solution to this problem is to aggregate over scales to arrive at a global measure of loss aversion. In our empirical study, we demonstrate and provide practical guidance on how to undertake calculations to that purpose. Our approach requires no consideration of scale prior to estimation, but it makes clear that scale is, in general, necessary for ex-post interpretation of loss aversion measures.

The framework we adopt in this paper is a familiar one. Following Tversky and Kahneman (1992), we define utility (or 'value') as a piecewise function governed by three parameters $\alpha, \beta$ and $\lambda$, where $\alpha$ and $\beta$ govern utility curvature in the gain and loss domains and $\lambda$ pivots utility at zero. It is common within this framework to impose the restriction that $\alpha=\beta$. The appeal of this restriction is that it simplifies the interpretation of $\lambda$, typically interpreted as an index of loss aversion. The importance of this restriction is apparent from the way in which Prospect Theory (PT) has been implemented and interpreted since Tversky and Kahneman (1992). For example, loss aversion as formulated by Köbberling and Wakker (2005) requires symmetry for the constant relative risk aversion (CRRA) utility function and if $\lambda$ is identified from a normalization restriction, then its value will be dependent on a point of normalization unless symmetry is imposed. From an econometric perspective, we argue 
that researchers should not automatically impose utility 'symmetry' within PT models just because of the apparent simplicity it affords in the interpretation of $\lambda$.

From a statistical perspective, there is no reason to impose symmetry without testing the restriction first. Indeed, there are studies that provide statistical evidence against symmetry. But, because of the issues surrounding the definition and interpretation of loss aversion, these results are often reported with strong caveats. For example Andersen et al. (2010) state:

"...there is a significant theoretical trade-off if one maintains this difference between $\alpha$ and $\beta$, stressed by Köbberling and Wakker (2005, Section 7), so this is not the sort of constraint that one should decide on purely empirical grounds." (p. 561).

Furthermore, rather than rejecting symmetry when it is inconsistent with the data, some researchers take a different stance. For example, Scheibehenne and Pachur (2015) support the imposition of the equality restriction;

"In our implementation of CPT, we set $\alpha=\beta$ because Nilsson et al. (2011) found that estimating $\alpha$ and $\beta$ separately can lead to a serious mis-estimation of $\lambda . "$ (p. 394).

Here, we take the view that symmetry should be tested empirically, not simply imposed a priori. Furthermore, if symmetry is not imposed, this does not preclude examining the extent of loss aversion, providing loss aversion is seen as being dependent on the size of prospect payoffs.

We propose an empirical approach that recognizes that, without symmetry, the identification of $\lambda$ requires a normalization method along with a point of normalization (a point made by Köbberling and Wakker, 2005). Specifically, as an alternative to imposing symmetry or the selection of a single normalization point (which is standard practice in the literature, as discussed by Abdellaoui et al., 2007), we propose and implement a method that averages over a weighted set of payoffs based on the use of a Gamma 'importance weighting' function. Our approach yields a "global" measure of loss aversion conditional on weightings selected by the researcher. As recognised in the literature, existing global measures of loss aversion offer attractive properties however, as observed by Abdellaoui et al. (2007) they have significant limitations when used empirically. As we demonstrate, size dependence is mitigated by our approach, but does not disappear altogether. In addition, our method also addresses the importance of 'scale' dependence in the evaluation of losses versus gains when symmetry of the utility function is not satisfied. This matters as numerous works that report degrees of loss aversion are divorced from the magnitude of the gains and lossses being compared. While some literature recognises this not to be a universal feature (e.g. Abdellaoui et al., 2007; p.1662), we believe that a clear articulation of this point is absent. For example, we do not find that Abdellaoui et al. (2007) 
clearly articulate that if a comparison of loss aversion parameters between studies is being made that researchers must use the same "real" point of comparison, not "nominal" values. However, they do note that numerous authors choose to evaluate risk aversion at different nominal values. Therefore, if the size conditionality of loss aversion was widely recognised, comparisons of loss aversion that ignore this feature would not be common within the literature. The issues we discuss are not confined to a particular utility function, but we specifically deal with two utility specifications: constant relative and constant absolute risk aversion (CRRA and CARA $)^{1}$.

This article therefore makes three points explicit that pertain to parametric estimates of loss aversion coefficients:

1) In general loss aversion coefficients are size dependent (i.e. the real size of the comparative gains and losses matters);

2) In a parametric context, size dependence can be avoided by assuming a symmetry condition but there is no other compelling reason to do so; and

3) Without a symmetry condition researchers must either pick a point (size) at which loss aversion is evaluated, or alternatively a range of points or some sort of "average" over these points.

Our recommendation is that researchers "average" over the size of payoffs, and do so using a Gamma weighting scheme, admitting that some size comparisons matter more than others, and making this judgement is unavoidable in all empirical contexts. In developing our approach, we make explicit the need for researchers to be aware about their normalization restrictions, and also be cognizant of the requirement that normalizations require real and not just nominal equivalence (of $\lambda$ estimates) in order to be comparable from one study to another. The applied literature frequently overlooks this requirement, while reporting parameter values that are taken to support 'loss aversion'.

Apart from the methodological contribution, our research adds to the literature that considers risk preferences in the field using PT (e.g. Tanaka et al., 2010; Liu and Huang, 2013; and Ward and Singh, 2015). We employ data from a survey of Ghanaian farmers. We estimate the data employing alternative utility specifications (CRRA and CARA) whilst allowing for heterogeneity amongst respondents by using a Hierachical Bayesian Logit (HBL). We also adopt a Bayesian log marginal likelihood (LML) approach to model discrimination using the HBL.

Our results unambiguously show that the CRRA is preferred to CARA, and 'symmetry' is rejected for the CRRA form (though not by the CARA). The values of $\lambda$ differ by utility specification, although our approach to integration of normalizations narrows the differences. Moreover, when we consider the implications for certainty equivalents, we find that the differences are actually quite small. The fact that we should not necessarily expect similar estimates of $\lambda$ when using alternative functional 
forms is also something we discuss.

\section{Loss Aversion: Definitions and Empirical Evidence}

\subsection{Theoretical Definitions}

Various definitions of loss aversion have been offered that relate to the behavior of an everywhere continuous and almost everywhere twice differentiable utility function $U(z)$ for which $z$ is usually some monetary amount (for a summary of the literature see Abdellaoui et al., 2007). These include a) $-U(-z)>U(z)$ for all $z>0$ (assuming $U(0)=0$ ); b) $U^{\prime}(-z)>U^{\prime}(z)$ (Wakker and Tversky, 1993) for all $z>0$; and c) discontinuity of the first derivatives of the utility function at zero, with the limits

of the derivatives from the left being larger than that from the right $\left(\lambda=\frac{\lim _{z \uparrow 0} U^{\prime}(z)}{\lim _{z \downarrow 0} U^{\prime}(z)}>1\right)$ (Köbberling and Wakker, 2005).

What these definitions and others illustrate is that there is no consensus as to what loss aversion technically means (Zank, 2010). Globally concave functions can satisfy properties a) and b) but whether the avoidance of losses is due to 'risk' rather than 'loss' aversion is a debatable point. On the other hand a utility function may satisfy property c) but have the reverse of a) and b) nearly everywhere. Also, a utility function can exist that has continuous second derivatives (thus satisfying property c) yet has most of the properties advocated by PT, i.e. being concave to the right of zero, convex to the left of zero, an inflexion point at zero, and exhibiting properties a) and b). Definition c) has compelling logic based on the idea that loss aversion pivots a well behaved basic utility function at zero. However, if this is accepted as the total manifestation of loss aversion, it has (as acknowledged by Köbberling and Wakker, 2005) few empirical consequences in terms of the restrictions on choices that a loss averse individual must make. ${ }^{2}$

The connection between the $\lambda$ parameter and loss aversion is not singular and direct. Clearly, $\lambda$, plays a key role in the evaluation of losses relative to gains and we will frequently refer to $\lambda$ as the loss aversion parameter or index. However, in doing so, we recognize that there are compelling reasons to argue that such a parameter need not always be viewed as the sole embodiment of loss aversion.

\subsection{Previous Empirical Evidence on Loss Aversion}

To date, the majority of empirical studies report values of $\lambda$ that tend to be greater than one though a minority report estimates of $\lambda<1$. For example, Booij et al. (2010) summarize a number of studies that all report $\lambda$ being greater than one, although several studies cited have point estimates close to one. Furthermore, Schmidt and Traub (2002) explicitly state in their conclusions that they find mixed evidence regarding loss aversion. There are also examples of studies that estimate low values of loss aversion. For example, Andersen et al. (2010) and Nilsson et al. (2011) both estimate PT specifications that are reasonably flexible and, in both cases, they not only report results that 
reject symmetry but also estimates of $\lambda<1$. However, both sets of results are reported in a very circumspect manner. For example, Nilsson et al. (2011) state the following regarding the results they report from their experimental data:

" the full version of CPT tend to underestimate $\lambda$ if $\alpha$ and $\beta$ are allowed to take different values. This possibility compromises the psychological interpretation of the model parameters." (p. 90).

Another important issue with the empirical literature relates to how prior expectations regarding the magnitude of loss aversion shape experimental designs. For example, Tanaka et al. (2010) employed a switching point experimental design that maintains the symmetry restriction as well as assuming that the loss aversion parameter is greater than one. While such designs may produce estimates that support loss aversion, they also ensure ex ante that $\lambda>1$.

A further issue within the empirical literature relates to the choice of functional forms. Many studies cite Stott (2006) who presents results relating to the relative performance of many common functional forms. Scott (2006) found that with regard to the utility function, CRRA specifications are unequivocally superior to alternative forms. Similar findings have also been reported by Balcombe and Fraser (2015). However, these findings pertained only to the gain domain. CRRA utility function specifications have also been employed in experimental studies in the risk literature (see Booij et al., 2010) as well as field experiments conducted with farmers in developing economies (e.g. Ward and Singh, 2015). At this point, little work has been done on optimal functional forms in mixed domain studies, and relatively little is known about how these impact estimates of $\lambda$.

Finally, there is a growing body of psychology literature that has begun to challenge the meaning of loss aversion. There are several studies that have shown that loss aversion need not be present in data depending on how an experimental design is implemented. Both Ert and Erev (2013) and Walasek and Stewart (2015) report results that show how manipulation of experimental designs can reveal loss aversion, loss neutrality and gain seeking behavior.

\section{Theoretical Properties and Empirical Interpretation}

\subsection{Piecewise Utility}

We begin by taking a piecewise utility function of the form, which nests that used by Tversky and Kahneman(1992)

$$
U\left(z, u^{+}, u^{-}, \lambda\right)=u^{+}(z) \text { for } z \geq 0 ; U\left(z, u^{+}, u^{-}, \lambda\right)=\lambda u^{-}(z) \text { for } z<0
$$

where $\lambda$ is a positive constant, $u^{+}(z)$ and $u^{-}(-z)$ are defined all $z \geq 0$ and are monotonically 
increasing over the whole range, $u^{+}(0)=-u^{-}(0)=0$ and $u^{+}(z)$ and $u^{-}(-z)$ are (finitely) twice differentiable everywhere on $z>0$. Next we observe that the piecewise ratio

$$
\tau_{U}(z)=\frac{-u^{-}(-z)}{u^{+}(z)} \text { for } z>0
$$

and the piecewise derivative ratio

$$
\gamma_{U}(z)=\frac{u^{-\prime}(-z)}{u^{+\prime}(z)} \text { for } z>0
$$

(observing that $\left.\tau_{U}^{\prime}(z)=\frac{u^{+\prime}(z)}{u^{+}(z)}\left(\gamma_{U}(z)-\tau_{U}(z)\right)^{3}\right)$ have played important roles in defining measures of loss aversion (Köbberling and Wakker, 2005) when at $\lambda=1$ symmetry holds where $\tau_{U}(z)=1$ for all $z>0$.

\subsection{Scaling of $\lambda$}

For any $U$ in equation (1), there exists a space $\left(\Omega_{U}\right)$ of equivalent utility functions $\tilde{U} \in \Omega_{U}$ defined by positive constants $\omega^{+}$and $\omega^{-}$such that $U\left(z, u^{+}, u^{-}, \lambda\right) \equiv \tilde{U}\left(z, \tilde{u}^{+}, \tilde{u}^{-}, \tilde{\lambda}\right)$, where $\tilde{u}^{+}=\omega^{+} u^{+}$ and $\tilde{u}^{-}=\omega^{-} u^{+}$such that $\tilde{\lambda}=\frac{\omega^{+}}{\omega^{-}} \lambda$. This mean we can always 'scale' $u^{+}, u^{-}$and $\lambda$ and retain the same preference ordering as $U$ (see also Wakker (2010), Observation 8.8.1). We will refer to $\tilde{U}$ as a 'scaled' version of $U$. Equally, we refer to $\Omega_{U}$ as the functional space generated by $U$, and $U$ as the basis function (or hereon "basis" to be succint) of $\Omega_{U}$ (that is the basis is an arbitrary choice of one function from a space of equivalent functions). Since all $\tilde{U} \in \Omega_{U}$ may have different values of $\tilde{\lambda}$ it makes no particular sense to assert that $\tilde{\lambda}$ should take a particular value, unless further conditions are placed on the space of functions.

\subsection{Functional Forms}

We adopt two functions are frequently employed in the literature. First, there is the CRRA form:

$$
u^{+}(z)=z^{\alpha} ; u^{-}(z)=-(-z)^{\beta}
$$

and second the CARA form:

$$
u_{1}^{+}(z)=\frac{1-e^{-\alpha z}}{\alpha} ; u_{1}^{-}(z)=-\frac{1-e^{\beta z}}{\beta}
$$

\subsection{Normalizations}

Köbberling and Wakker (2005) observe that Tversky and Kahneman (1992) adopt an implicit condition that $\tilde{u}^{+}(1)=-\tilde{u}^{-}(1)$. This normalization that identifies $\tilde{\lambda}$ means that having specified a $U$, another condition is used to identify a particular $\tilde{U}$ within $\Omega_{U}$ (and therefore $\tilde{\lambda}$ ). Thus, 
$\tilde{u}^{+}(1)=-\tilde{u}^{-}(1)$ implies that $\tilde{\lambda}=\lambda$ for the CRRA form adopted by Tversky and Kahneman (1992). Alternatively, Tversky and Kahneman (1992) could be viewed as adopting the convention that $\tilde{u}^{+\prime}\left(\left(\alpha \beta^{-1}\right)^{\frac{1}{\beta-\alpha}}\right)=-\tilde{u}^{-\prime}\left(\left(\alpha \beta^{-1}\right)^{\frac{1}{\beta-\alpha}}\right)$. What needs to be recognized in general is that without normalizations $\lambda$ remains unidentified unless symmetry is imposed. If symmetry holds these normalizations hold for all $z>0$, thus highlighting the convenience of the symmetry restriction. In effect, symmetry frees the identification of $\lambda$ from both the type of normalization or the value $(z)$ at which the normalization is imposed.

Köbberling and Wakker (2005) also propose a normalization which amounts to imposing $\tilde{u}^{+\prime}(0)=$ $-\tilde{u}^{-1}(0)$, if these derivatives exist, or a lesser condition if they do not. If we require the piecewise utilities or the piecewise derivatives to be equal at some point, equally left or right of zero, then $\tilde{\lambda}$ will be the same for any scaled equivalent utility function that obeys that condition.

While attractive at a philosophical level, the Köbberling and Wakker (2005) normalization only has empirical consequences in the neighborhood of zero (Harrison and Swarthout, 2016). As a result, it follows that different utilities can be practically identical except in the empirically negligible neighborhood of zero, but can either be very loss averse in the Köbberling and Wakker (2005) sense, or have no loss aversion at all. We see no problem with researchers reporting the Köbberling and Wakker (2005) measure (if it exists) as their index of loss aversion, if they wish. But, it is informative to report the value of $\tilde{\lambda}$ that would be derived under normalizations $\tilde{u}^{+}(z)=-\tilde{u}^{-}(z)$ or $\tilde{u}^{+\prime}(z)=-\tilde{u}^{-\prime}(z)$ for different values of $z$.

Importantly, normalizations require no decision about scaling prior to estimation. For any given specification of $u$, the parameters can be estimated first and the scaling convention can then be imposed by calculating $\tilde{\lambda}=\lambda \frac{-u^{-}(z)}{u^{+}(z)}$ (or alternatively $\left.\tilde{\lambda}=\lambda \frac{u^{-1}(z)}{u^{+\prime}(z)}\right)$ where $\lambda, u^{-}(z)$ and $u^{+}(z)$ specify $\Omega_{U}$. In practice, the symmetry condition (implicitly) imposed by many researchers requires that $\frac{-u^{-}(z)}{u^{+}(z)}=1$ for all $z>0$, thus $\tilde{\lambda}$ is normalization invariant. Importantly, however, it should be recognized that, without symmetry, the value of $\lambda$ that generates $\Omega_{U}$ need not correspond with a normalized $\tilde{\lambda}$. For example, if a CARA functional form is specified, then $\frac{-u^{-}(z)}{u^{+}(z)}=1$ will not hold for any point $z$, and both need to be scaled at some point of normalization. The fact that this is important is simply illustrated if researchers estimate $\lambda$.

Consider the following example. If one researcher uses the CARA form as their basis and another an exponential form $\left(u_{2}^{+}(z)=1-e^{-\alpha z}\right.$ and $\left.u_{2}^{-}(z)=-\left(1-e^{\beta z}\right)\right)$, it is evident that providing $\alpha$, $\beta>1$ then $\lambda_{2}=\frac{\alpha}{\beta} \lambda_{1}$. If they can agree to a scaling condition, (for example, $u^{+}(1)=-u^{-}(-1)$, or $\tau(1)=1)$ then for this scaling convention they will agree that both will have to scale their functions since neither of them obey this condition unless $\alpha=\beta$. We note that under this scaling convention 
for the CARA form, the researcher could choose to scale as $\frac{\omega_{1}^{-}}{\omega_{1}^{+}}=\frac{\beta\left(1-e^{-\alpha}\right)}{\alpha\left(1-e^{-\beta}\right)}$ and the exponential form as $\frac{\omega_{2}^{-}}{\omega_{2}^{+}}=\frac{\left(1-e^{-\alpha}\right)}{\left(1-e^{-\beta}\right)}$. Since $\lambda_{2}=\frac{\alpha}{\beta} \lambda_{1}$ they agree as follows: $\tilde{\lambda}=\frac{\alpha\left(1-e^{-\beta}\right)}{\beta\left(1-e^{-\alpha}\right)} \lambda_{1}=\frac{\left(1-e^{-\beta}\right)}{\left(1-e^{-\alpha}\right)} \lambda_{2}$. Finally, we note that it is under the CARA form that Köbberling and Wakker (2005) justify the use of $\lambda$.

\subsection{Identifying $\tilde{\lambda}$ under Asymmetry using Importance Weightings}

If symmetry is not imposed then the identification of $\tilde{\lambda}$ requires the selection of a normalization point or set of normalization points. We propose that choosing these values is relatively straight forward if one accepts that some levels of payoffs are more important than others when evaluating loss aversion. For example, for most UK citizens, considering the range of prospects between $\pm £ 10$ and $\pm £ 100,000$ is practically more important than between $\pm £ 1$ million and $\pm £ 10$ million. Accordingly, we propose to adopt an 'importance weighting function' $f(z)$ that assigns greater importance to some values $(z)$ relative to others and then computes a weighted average over normalized values of $\tilde{\lambda}(z)$, constructed from basis utility $U$ defined by $\lambda$.

In what follows define $\ln (\tilde{\lambda}(z))=\ln (\lambda)+\ell(z)$ where $\ell(z)$ can be defined in either of two ways:

$$
\ell(z)=\ln \left(\tau_{U}(z)\right) \text { or } \ln \left(\gamma_{U}(z)\right)
$$

(where $\ln \left(\tau_{U}(z)\right)$ is defined in equation 2 and $\ln \left(\gamma_{U}(z)\right)$ in equation 3 ). We then define the measure $\Lambda$, where

$$
\Lambda=\exp \int_{0}^{\infty} \ln (\tilde{\lambda}(z)) d z=\lambda \exp \left(\int_{0}^{\infty} \ell(z) f(z) d z\right)
$$

where $f(z)$ is an 'importance function' with the property that $\int_{0}^{\infty} f(z) d z=1$. Thus, $\Lambda$ is a 'weighted mean' of different normalization measures $\lambda(z)$ where different levels of importance are assigned to $z$.

Given a choice of $f(z), \Lambda$ can be calculated by simulation, or by closed form approximations (which in some cases are exact). Under symmetry, no such calculation is required since $\Lambda$ collapses to $\lambda$. The use of a Gamma distribution for the importance function $f(z)$ is one obvious possibility, since it only bounded below by zero, and provides a flexible range of possible weightings. It also has the attractive property that it provides a simple solution in terms of approximations.

The Gamma distribution $f(z, k, j)$ can be fully defined by setting the mode equal to $m$ and the mean equal to $\mu(m<\mu)$, in which case we have

$$
f(z, k, j)=\frac{1}{\Gamma(k) j^{k}} z^{k-1} e^{-\frac{z}{j}} \text { where } j=\mu-m ; k=\frac{\mu}{\mu-m}
$$


The Gamma distribution has the property that

$$
h_{n}=\int_{0}^{\infty} z^{n} f(z, k, j) d z=\frac{\Gamma(k+n) j^{n}}{\Gamma(k)}
$$

If $\ell(z)$ is approximated using a second order expansion (see Appendix C for full details) around the mean of the importance function then we can show that

$$
\begin{aligned}
\Lambda \simeq & \lambda\left(\int_{0}^{\infty} \ell(z) f(z) d z\right) \\
& \text { where } \\
\left(\int_{0}^{\infty} \ell(z) f(z) d z\right)= & \exp \left(\ell(\mu)-\ell^{\prime}(\mu) \mu+\frac{\ell^{\prime \prime}(\mu)}{2} \mu+\left(\ell^{\prime}(\mu)-\ell^{\prime \prime}(\mu) \mu\right) h_{1}+\frac{\ell^{\prime \prime}(\mu)}{2} h_{2}\right)
\end{aligned}
$$

This approximation is accurate for a wide range of parameter values.

In the case where we have two functional forms (CRRA and CARA), combined with two alternative ways of defining $\ell(z)$ (as in [6]), this yields four possible definitions of $\ell(z)$ (which in turn gives four definitions for $\Lambda$ ). Table 1 summarizes the definitions of $\ell(z)$ by functional form (in rows) and normalization type (in columns) along with the approximation for $\left(\int_{0}^{\infty} \ell(z) f(z) d z\right)$ in equation [10] which we denote more succinctly as $\int \ell f$. Readers are reminded that under symmetry $\Lambda \equiv \lambda$.

Table 1: Empirical Bounded Measures of Loss Aversion

\begin{tabular}{ccc}
\hline & Normalization "=Utility" & Normalization "=MU" \\
CARA & $\ell(z)=\ln \left(\frac{\alpha}{\beta} \frac{1-e^{-z \beta}}{1-e^{-z \alpha}}\right)$ & $\ell(z)=\ln \left(\frac{e^{-z \beta}}{e^{-z \alpha}}\right)$ \\
& $\int \ell f=\Pi$ & $\int \ell f=(\alpha-\beta) h_{1}$ \\
CRRA $\quad \ell(z)=\ln \left(\frac{z^{\beta}}{z^{\alpha}}\right)$ & $\ell(z)=\ln \left(\frac{\beta}{\alpha} \frac{z^{\beta}}{z^{\alpha}}\right)$ \\
$\ln (\mu)-\frac{3}{2}+2 \frac{h_{1}}{\mu}-\frac{1}{2} \frac{h_{2}}{\mu^{2}}, \Pi \simeq c_{0}+c_{1} h_{1}+c_{2} h_{2}\left(\right.$ see Appendix C for $c_{0}, c_{1}$ and $\left.c_{2}\right)$ and $h_{n}$ \\
$=\frac{\Gamma\left(\frac{\mu}{\mu-m}+n\right)(\mu-m)^{n}}{\Gamma\left(\frac{\mu}{\mu-m}\right)}$.
\end{tabular}

Importantly, the approach we have introduced does not depend on the denomination used to specify the ordering, provided it is recognized that to be comparable and consistent, real rather than nominal points or normalization are specified. ${ }^{4}$

\section{The Econometric Framework}

Our framework describes choice with a random utility model that embeds the deterministic PT model. Thus, the utility $U_{i j s}$ is for the $j^{\text {th }}$ person $(j=1, \ldots, J)$ in the $i^{\text {th }}$ option $(i=1, \ldots, N)$ from the $s^{t h}$ choice prospect $(s=1, \ldots, S)$ is: 


$$
U_{i j s}=\mathcal{F}\left(x_{i j s}, \theta_{j}\right)+e_{i j s}
$$

where $x_{i j s}$ is a $(K \times 1)$ vector and $\theta_{j}$ is a vector of parameters characterizing the systematic preferences of an individual. $\mathcal{F}\left(x_{i j s}, \theta_{j}\right)$ is defined here as being proportional to the certainty equivalent of a prospect. We also define: $y_{i j s}=1$ if $U_{i j s}=\operatorname{Max}\left(U_{1 j s}, \ldots, U_{N, j, s}\right)$. The assumption of an extreme value error $e_{i j s}$ that is independent across, $i, j$ and $s$ implies the probability of choosing option $i$ for the $j^{\text {th }}$ person in the $s^{\text {th }}$ choice prospect is (where $\beta_{j}$ is known):

$$
p_{i j s}\left(\beta_{j}\right)=\frac{e^{\mathcal{F}\left(x_{i j s}, \theta_{j}\right)}}{\sum_{i} e^{\mathcal{F}\left(x_{i j s}, \theta_{j}\right)}}
$$

We model respondent heterogeneity by employing a HBL specification such that a prior distribution is assigned to the latent parameters $\theta_{j}$. When employing a HBL specification it is assumed that $\theta_{j}$ is multivariate normal such that transformations can be used to bound the parameters into particular regions or give them alternative distributions. The bounds for all parameters were implemented by a transformation of normals approach:

$$
\theta=L b+(U b-L b)\left(\frac{e^{\kappa}}{1+e^{\kappa}}\right)
$$

where $L b$ is the lower bound, $U b$ is the upper bound and $\kappa$ is a random variate with normal prior with mean zero and variance of one. This constitutes a fairly diffuse prior within the bounds but with a mean at the center. For further detail about estimation of the HBL, readers are referred Chapter 12 of Train (2009), where the algorithms and related GAUSS code employed to simulate posterior distributions are described in detail.

In keeping with the PT literature, we refer to $x_{i j s}$ as a prospect were $x_{i j s}^{\prime}=\left(p_{i j s}^{\prime}, z_{i j s}^{\prime}\right)$ and $p_{i j s}$ is a vector of probabilities (summing to one) and $z_{i j s}$ is a vector of associated payoffs. The key elements of a random utility implementation of a PT model are: the utility (value) function $(U)$; the weighting function $(w)$; and the link function $(l)$.

Our exposition will be limited to the case of two payoffs. Accordingly, denote each payoff as $z_{i j s t}$, $t=1,2$ with $z_{i j s}^{\prime}=\left(z_{i j s 1}, z_{i j s 2}\right)$ and define the functions

$\operatorname{ord}\left(z_{i j s t}\right)=1$ if $z_{i j s t}$ is the higher payoff (over $\left.t=1,2\right)$ and $\operatorname{ord}\left(z_{i j s t}\right)=0$ otherwise $\operatorname{sgn}\left(z_{i j s t}\right)=1$ if $z_{i j s t}$ is positive and $\operatorname{sgn}\left(z_{i j s t}\right)=0$ otherwise 
We define utility in a piecewise form:

$$
U_{j}\left(z_{i j s t}, \operatorname{sgn}\left(z_{i j s t}\right)\right)
$$

Next, probability weightings are decided according to both order and sign of the payoffs. They take the form $w_{j}\left(p_{i j s t}, \operatorname{ord}\left(z_{i j s t}\right), \operatorname{sgn}\left(z_{i j s t}\right)\right)$ using two functions $w_{+}($.$) and w_{-}($.$) (defined parametrically$ later on) as

$$
\begin{aligned}
w_{j}\left(p_{i j s t}, 1,1\right) & =w_{j}^{+}\left(p_{i j s t}\right), w\left(p_{i j s t}, 0,1\right)=1-w_{j}^{+}\left(p_{i j s t}\right) \\
w_{j}\left(p_{i j s t}, 0,1\right) & =w_{j}^{-}\left(p_{i j s t}\right), w\left(p_{i j s t}, 0,0\right)=1-w_{j}^{-}\left(p_{i j s t}\right)
\end{aligned}
$$

The way in which order effects this function differs between the gain and loss domain, such that the PT function calculates utility as

$$
U^{*}\left(x_{i j s}, \theta_{j}\right)=\sum_{t=1}^{2} w_{j}\left(p_{i j s t}, \text { ord }\left(z_{i j s t}\right), \operatorname{sgn}\left(z_{i j s t}\right)\right) U\left(z_{i j s t}, \operatorname{sgn}\left(z_{i j s t}\right)\right)
$$

from which we can calculate a certainty equivalent measure $\xi\left(x_{i j s}, \theta_{j}\right)$ from $U^{*}\left(x_{i j s}, \theta_{j}\right)$.

We employ two utility specifications, the CRRA

$$
u_{j}^{+}\left(z_{i j s t}\right)=z_{i j s t}^{\alpha_{j}} ; u_{j}^{-}\left(z_{i j s t}\right)=-\lambda_{j}\left(-z_{i j s t}\right)^{\beta_{j}}
$$

and the CARA

$$
u_{j}^{+}\left(z_{i j s t}\right)=\frac{1-\exp \left(-\alpha_{j} z_{i j s t}\right)}{\alpha_{j}} ; u_{j}^{-}\left(z_{i j s t}\right)=-\lambda_{j} \frac{\left(1-\exp \left(\beta_{j} z_{i j s t}\right)\right)}{\beta_{j}}
$$

where $\alpha_{j}, \beta_{j}$, and $\lambda_{j}$ are positive numbers. The values of $\alpha_{j}$ and $\beta_{j}$ govern curvature within the gain and loss domains respectively (for more details see Appendix E, Table 1E).

\subsection{Probability Weighting}

For our probability weighting functions, we employ the Prelec II function

$$
w_{j}^{+}\left(p_{i j s t}\right)=e^{\left(-\gamma_{j}\left(-\ln \left(p_{i j s t}\right)\right)^{\delta_{j}}\right)} ; w_{j}^{-}\left(p_{i j s t}\right)=e^{\left(-\epsilon_{j}\left(-\ln \left(p_{i j s t}\right)\right)^{\eta_{j}}\right)}
$$

This function delivers a non-stochastic predictor of utility of prospects using (16). This is a flexible functional form that can capture "inverse-S" behavior, it can be concave or convex over the range of probabilities and the parameter values in the loss and gain domain can differ. Support for this choice 
of weighting function is given by Balcombe and Fraser (2015) with the data from Stott (2006).

\subsection{Probability Linkage}

Our framework needs to be embedded within a stochastic framework whereby it is used as a probable predictor of choice. The 'link' function plays this role and we employ the logit form . This, however, does not fully specify the nature of the link. A common approach has been to equate $\mathcal{F}()=.\phi u($.$) in (16). However, we prefer to use the certainty equivalent \mathcal{F}()=.\phi \xi($.$) , the reason$ being that under the utility specification $\lambda$ plays a dual role in determining the relative strength of the signal as well as the ordering or mixed gambles. Therefore, we specify

$$
U_{i j s}=\phi_{j} \xi\left(x_{i j s}, \theta_{j}\right)+e_{i j s}
$$

Under this condition, and the assumptions above, it is the difference in certainty equivalence that drives the non-stochastic element of choice between prospects, since the probability of choosing $x_{i j s}$ over $x_{i^{*} j s}$ becomes

$$
p=\frac{1}{1+\exp \left(\xi_{j}\left(u\left(x_{i j s}, \theta_{j}\right)-\xi\left(x_{i^{*} j s}, \theta_{j}\right)\right)\right)}
$$

\subsection{Parameter Interpretations, Priors and Restrictions}

When discussing $\mathrm{PT}$, we distinguish between the over-arching structure of $\mathrm{PT}$, and restrictions placed on that structure in accordance with the type proposed by Tversky and Kahneman (1992), allowing us to make the distinction between "strong" and "weak" versions of PT. Weak PT, as we define it, has the structure outlined above (see Chateauneuf and Wakker, 1999) without any particular restrictions on the shapes of the utility functions or probability transformations. Weak PT has reference point dependence around which the curvature of the utility functions may differ and probability weightings that can diverge from linearity. Stronger versions of PT, on the other hand, inherit the weak structure but might include additional restrictions on the nature of this structure, such as: concavity of the utility function in the gain domain; convexity of the utility function in the loss domain; a heavier weighting of losses to gains in terms of the utility function; and, inverse-S (IS) probability weightings (in both gain and loss domains). ${ }^{5}$

For estimation, we adopted a bounded approach to specifying the permissibly regions for each of the parameters which encompassed the vast majority of estimates in the preceding literature. For the CRRA utility parameters, we specified $\alpha, \beta \in(0.01,2)$ and for the CARA, we specified $\alpha, \beta \in(-1,1)$. Given the scaling of the data, these regions allow for quite extreme risk aversion and/or seeking behavior in terms of the utility functions. For the Prelec II parameters, we specified $\delta, \gamma, \varepsilon, \eta \in(0.4,1.6)$ which obviously includes the linear case (i.e., expected utility) but allows quite 
large divergences in behavior. For $\lambda$, we specified $\lambda \in(0.5,3)$ which include the vast majority of estimates found in the literature. As discussed previously, this parameter is denomination dependent, but our estimate has an implicit normalization "equal utility" at one (a small gamble in the context of this study), and would be expected therefore to be consistent with much larger values at higher normalization points. Finally, the parameter $\phi$ was specified as a log-normal distribution and thus contained no upper bound. ${ }^{6}$ A summary of how to interpret the parameters estimated is provided in Appendix E.

\section{The Risk Survey}

\subsection{Descriptive Statistics}

Our study was undertaken in the central region of Ghana involving 384 food crop farmers selected using a multi-stage random sampling technique. In the first stage, six districts in the central region were selected at random. Next eight villages were randomly selected in each region and finally within each village eight farmers were sampled based on lists provided by local agricultural extension officers. Data was collected during face-to-face interviews by trained field assistants from the University of Cape Coast and agricultural extension officers from the Ghanaian ministry of agriculture between May and July 2013. The survey was composed of two parts: a general survey that examined aspects of agricultural production activity; and a specific risk elicitation component. In addition, the survey collected descriptive statistics that are reported in Table A1 in Appendix A.

\subsection{Experimental Design Procedure}

The risk elicitation component of the survey employed an approach designed so as to minimize implementation and cognitive difficulty in keeping with similar designs that have analyzed risk/loss aversion attitudes of farmers in the field (i.e. Booij et al., 2010). We constructed a series of choice tasks (i.e. investment alternatives) with two prospects, A or B, that had monetary outcomes, along with associated probabilities. The prospect pairs were generated with a range of probabilities, and outcomes ranging from 0 to 10 in the positive domain and 0 to -10 in the negative domain. All possible 'two payoff' prospects were generated, and all pairs for which one prospect first-order stochastically dominated another were eliminated. The remaining pairs were next classified by examining the difference in the utilities under EU assuming a CRRA utility function. All gain (and loss) domain prospect pairs were ordered according to a "change point" whereby a switch would be made from one prospect to another for a given CRRA parameter ranging from 0.5 to 1.25 . We then selected prospect pairs periodically as we moved through the ordered pairs with a selection frequency that gave us 16 pairs in each of the gain, loss and mixed domains. The mixed domain pairs were ordered, but not according to the curvature in the utility, but instead where we observed a switch in the "loss aversion" 
parameter $\lambda$ (range 0.5 through to 2). Here we assumed the curvature in Tversky and Kahneman (1992), $\alpha=0.88$ and $\beta=0.88$. Finally, the payoffs were scaled by a factor of GH $\phi 100$ (Ghana Cedis) before implementation in the field. This was done to bring the prospects closer to the real world situation in which the sampled farmers find themselves. Most farmers would deem a loss or gain of $\mathrm{GH} \not 100$ to be moderate or small, whereas a loss or gain of $\mathrm{GH} \not 1000$ would have considerable consequences for most farmers.

\subsection{The Elicitation Method}

The risk elicitation part of the survey instrument was divided into three parts: i) prospects in the gain domain; ii) prospects in the loss domain; and iii) prospects in the mixed domain. In the experiment, each farmer undertook a total of 32 choice tasks from one of two versions of the survey instrument. In one version farmers were offered eight sets of paired prospects with positive outcomes (out of 16), eight sets of paired prospects with negative outcomes (out of 16), and 16 sets of paired prospects involving mixed outcomes. The second version employed the remaining eight positive and loss domain prospects but re-used the 16 mixed domain prospects. The final set of all prospect pairs are shown in Appendix B.

The experiment was explained in a face-to-face setting with farmers. The prospects were presented in terms of probabilities of drawing green or blue marbles from a bag. Each farmer was made to go through three practice tasks to be sure that they understood the experiment before the actual 32 experimental tasks were introduced.

Probabilities were demonstrated to farmers by employing a ball drawing randomization approach using colored balls. Two bags of different colored balls (Green or Blue) were prepared in advance. Farmers understood that each bag represented one of the prospects (investment alternatives they had to choose from). For each prospect choice farmers were required to draw balls from the bags they would choose in order to determine how much they received or lost as outcomes from their investment decisions in the upcoming season. The proportions of different colored balls represented the probabilities of the prospect outcomes if a farmer decided to play either Green prospect (Prospect A) or Blue prospect (Prospect B).

Each choice task was explained to the farmers as follows:

- "You have an opportunity to decide which prospect A or B you invest your limited amount of money as you enter the coming season".

- "Prospect A is represented here with a Green bag and Prospect B is represented with a Blue bag". 
- "The Green bag contains 10 balls. 6 of the 10 balls are white in color and the remaining 4 of the balls are green colored. If you decide to choose this Green bag and randomly draw any of the green balls from it, you will receive GH 4900 as profit on your investment. If instead you draw any of the white balls from it, you will receive nothing."

- "The Blue bag also contains 10 balls all of which are blue in color. If you decide to choose this Blue bag and draw any blue ball, you will receive GH $\not 100 . "$

- "For whichever bag you choose, you can only draw one ball."

- "Which of the two bags (Green or Blue) would you choose?"

The example described is shown in Figure 1:

\section{\{Approximate Position of Figure 1\}}

This experiment was repeated until all 32 tasks had been answered. To incentivize each farmer, they were told at the start of the experiment that one of their choices would be selected at random and they would receive the associated payoff (though payoffs would be proportional not actual amounts). This was done on an individual basis (different lotteries were selected for different farmers). No payments were made within a village until all the experiments had been conducted. We did not make negative payoffs binding, but simply to made no payoff at all if the lottery selected returned a negative payoff. This approach is similar to that employed by Ward and Singh (2015).

The payoff incentives given were in the form of soaps (GHф25), candles and matches (GH 415$)$, and candies $(\mathrm{GH} \not 10)$. These were reasonably generous incentives given that the minimum wage in Ghana in 2013 was GH $\not 5.24 .^{7}$ The incentives corresponded to high (10, 9 and 8), medium (7, 6, 5 and 4) and low payoffs (3,2 and 1). Finally, to ensure that no farmer got nothing for participation in the survey, each was given a small incentive (i.e., GH $\not 10)$ after the interview, even if they won nothing for the actual prospect selected. ${ }^{8}$

\section{Empirical Results}

We begin by examining relative model performance to identify which model specification is the best performing using logged marginal likelihoods (LMLs) following the method outlined in Balcombe and Fraser (2015). Details on model performance with regard to convergence and posterior predictions are reported in Appendix F. We then examine the resulting model estimates so as to assess various aspects of PT (EU). We pay particular attention to the loss aversion parameter, with and without symmetry, using the importance weighted approach. Finally, we examine the probability weightings in the gain, loss and mixed domains. ${ }^{9}$ 


\subsection{Model Performance and Risk Parameters}

The LMLs are presented in Table 2. Eight models were estimated, four of each utility type. We also tested three nested specifications for each of these value specifications: a test for symmetry; a test for linearity in the probability weightings as would be the case under EU; and the choice between the Prelec I and Prelec II probability weighting functions. Importantly, the LML can be employed in both a nested and non-nested context and thus can be used to compare models row wise or column wise.

Table 2. Log Marginal Likelihoods for Competing Models

\begin{tabular}{lcc}
\hline & CRRA & CARA \\
\hline M1:Asymmetric $(\alpha \neq \beta)+$ Prelec II & $-6097^{*}$ & -6241 \\
M2:Symmetry $(\alpha=\beta)+$ Prelec II & -6118 & $-6215^{*}$ \\
M3:Asymmetric $(\alpha \neq \beta)+$ Prelec I $(\delta=\eta=1)$ & -6137 & -6258 \\
M4:Asymmetric + Linear Probability $(\delta=\eta=\gamma=\varepsilon=1)$ & -6359 & -6324 \\
\hline \multicolumn{2}{c}{ Note:* indicates the preferred model in each column }
\end{tabular}

From Table 2, we can see that a comparison of columns indicates that nearly every CRRA specification is preferred to its corresponding CARA specification. Under equal prior odds, the posterior odds of any given model relative to another could be calculated by employing the $\mathrm{e}^{\text {Ratio }}$ where the Ratio is the ratio of two models log likelihoods. According to this criteria there is a categorical unambiguous preference for the CRRA M1 model which in effect has a posterior odds close to one relative to any other model. This means that if we conducted a model averaging exercise across all the models estimated, this model would simply be attributed all of the weight.

A comparison of rows shows that for the CRRA function the preferred specification is the most general model M1 which does not impose symmetry or reduce the flexibility of the probability weightings. The CARA results are similar with the exception that the symmetry restriction (M2) cannot be rejected for this model. For both CARA and CRRA forms the worst performing model by far is the one without probability weightings (M4). Therefore, in this respect our results are consistent with the majority of empirical work on PT which support the use of probability weightings. However, the rejection of symmetry for CRRA is important in the light of our discussion regarding loss aversion, since the evaluation of normalized values $\lambda(z)$ become point dependent, which we consider.

In Table 3, we give a summary of the parameter distributions for the preferred specifications for the CRRA and CARA forms. We have done this because empirical research frequently uses the CARA form and, therefore, it is interesting to contrast the findings with that of the CRRA function, even though for our data the CRRA form is categorically preferred. Importantly, these parameter 
estimates include the distribution across all respondents and thus reflect respondent heterogeneity. What are reported are the means, medians, and standard deviations for the parameter estimates for each individual generated from the HBL model.

Table 3: Parameter Estimates \& Distributions for Preferred Specifications

\begin{tabular}{lcccccccc}
\hline & $\alpha$ & $\beta$ & $\gamma$ & $\delta$ & $\varepsilon$ & $\eta$ & $\lambda$ & $\phi$ \\
\hline Mean & & & & & & & & \\
CRRA & 0.50 & 0.70 & 0.58 & 0.88 & 0.66 & 1.21 & 0.76 & 1.74 \\
CARA $*$ & 0.72 & 0.72 & 0.68 & 1.11 & 1.15 & 0.86 & 1.58 & 0.98 \\
\hline Stdv & & & & & & & & \\
CRRA & 0.22 & 0.11 & 0.03 & 0.05 & 0.07 & 0.16 & 0.25 & 0.37 \\
CARA & 0.39 & 0.39 & 0.11 & 0.10 & 0.04 & 0.25 & 0.72 & 0.32 \\
\hline Median & & & & & & & & \\
CRRA & 0.44 & 0.68 & 0.57 & 0.89 & 0.64 & 1.25 & 0.69 & 1.68 \\
CARA & 0.84 & 0.84 & 0.65 & 1.10 & 1.15 & 0.79 & 1.44 & 0.93 \\
\hline
\end{tabular}

Note:* Estimates are for the preferred specification (Symmetry is imposed for CARA)

In Table 3 for the CRRA form, we obtain mean estimates of $\bar{\alpha}=0.50$ and $\bar{\beta}=0.70$ which imply a tendency to have greater concavity in the gain domain relative to the convexity in the loss domain. These results yield support for the general contention of PT that utility is generally 'gain concave' and 'loss convex' (i.e. risk averse in gains and risk seeking in losses) which is also consistent with Wakker (2010, see pp.264 and 269). The CARA estimates, imply much greater 'gain concavity' and 'loss convexity' in the utility function.

Turning to loss aversion, we note that for the interpretation of the results our data has been scaled so that the smallest gambles are 1 and largest are 10 in the gain domain, and -1 and -10 in the loss domain. This is relevant to both the normalized importance weighted values of $\lambda$ (or $\Lambda$ ) that we subsequently report, but also for the interpretation of the $\alpha$ and $\beta$ which are denomination dependent for the CARA case.

The value of $\phi$ (signal to noise ratio) is generally greater for the CRRA function than for the CARA function which is consistent with the fact that the CRRA function is the better performing model. A value of $\phi$ translates a given signal in terms of the certainty equivalent into a probabilistic measure of choice. Therefore, a higher value of $\phi$ for the CRRA function indicates that the signal from the deterministic model generated under the CRRA specification is stronger.

With regard to probability weightings, we note the substantive difference between the probability weighting functions under CRRA and CARA utility. This supports and draws attention to the 
findings of previous studies (e.g. Balcombe and Fraser, 2015) that show how the findings with respect to one 'aspect' (e.g. probability weightings) can be subject to the choice of other aspects (e.g. the utility function). We present the probability weighting functions in Figure 2 for our preferred model (CRRA M1).

\section{\{Approximate Position of Figure 2\}}

These plots in Figure 2 are the probability weighting curves for all 384 respondents so as to give a general illustration of the average but also range of behaviors across individuals. The panels are for the gain and loss domain respectively, for the 'greater gain' $w_{j}^{+}(p)$ and larger loss' $w_{j}^{-}(p)$. The results indicate that the characteristic inverse-S shape is supported for all individuals in the gain domain. The corresponding inverse-S shape for the loss domain is supported for the majority of individuals. There is another group who are pessimistic in their attitudes towards largest loss (those above the 45 degree line).

\subsection{Importance Weighting Functions and Loss Aversion}

We now examine how our estimates of loss aversion are impacted by the use of the importance weighting functions. To do this we investigated the impact of alternative normalizations on the estimates of $\Lambda$ and for this purpose we also include the results for the CARA case where symmetry has not been imposed. The importance weightings we investigate are plotted in Figure 3.

\section{\{Approximate Position of Figure 3\}}

In each case we take the modal value of one (corresponding to the lowest payoff in our gamble set), but we experiment with three alternatives for the mean of the Gamma function $(\mu=5,10,20)$. Here, $x=10$ corresponds to the largest payoff in our gamble set $\mathrm{GH} \not{ }^{\prime} 1000$. The middle value $(\mu=10)$ yields our preferred importance function. It reflects our observations that $x=1$ (GH $\not 100)$ is considered small in terms of assessment by respondents, while a payoff of $\mathrm{GH} \not \mathbf{1 0 0 0}$ reflects a large payoff to respondents (GH $\not 1000$ or around $\$ 200$ US dollars). To put this in perspective, for Ghanian farmers, this corresponds to around one years annual income, which for the purposes here we would characterise as "large" though it may not be considered "large" in high income countries, or indeed for urban wage earners in Ghana ${ }^{10}$.

The importance function for $\mu=10$, gives approximately $25 \%$ of the weighting mass to values

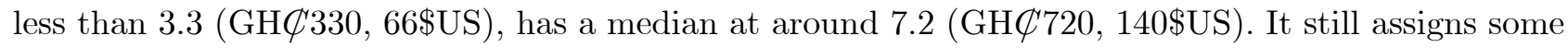
importance to values of 20 or 30 (which may arise in some investment decisions) but very little beyond.

How the choice of the mean of the Gamma function within the importance weighting function impacts estimates of loss aversion is summarised in Table 4. 
Table 4: Distributions of $\lambda$ and $\Lambda$ across respondents

\begin{tabular}{lllllllll}
\hline CRRA & Mean & Std & Min & $\mathbf{2 5 \%}$ & Median & $\mathbf{7 5 \%}$ & Max & Prop $>\mathbf{1}$ \\
\hline$\lambda$ Symmetrical & 0.98 & 0.13 & 0.82 & 0.90 & 0.95 & 1.01 & 1.54 & 0.28 \\
$\lambda$ Basis & 0.76 & 0.25 & 0.55 & 0.63 & 0.69 & 0.76 & 1.84 & 0.08 \\
$\Lambda$ Importance Weighted $(\mu=5)$ & 0.92 & 0.13 & 0.76 & 0.84 & 0.88 & 0.95 & 1.49 & 0.18 \\
$\Lambda$ Importance Weighted $(\mu=10)$ & 1.06 & 0.09 & 0.84 & 0.99 & 1.04 & 1.11 & 1.37 & 0.71 \\
$\Lambda$ Importance Weighted $(\mu=20)$ & 1.21 & 0.12 & 0.81 & 1.15 & 1.21 & 1.29 & 1.71 & 0.95 \\
\hline CARA & Mean & Std & Min & $\mathbf{2 5 \%}$ & Median & $\mathbf{7 5 \%}$ & Max & Prop $>\mathbf{1}$ \\
\hline$\lambda$ Symmetrical & 1.58 & 0.72 & 0.61 & 0.95 & 1.44 & 2.12 & 3.00 & 0.72 \\
$\lambda$ Basis & 1.63 & 0.46 & 0.79 & 1.21 & 1.61 & 2.03 & 2.66 & 0.92 \\
$\Lambda$ Importance Weighted $(\mu=5)$ & 1.80 & 0.78 & 0.81 & 1.23 & 1.63 & 2.10 & 5.57 & 0.92 \\
$\Lambda$ Importance Weighted $(\mu=10)$ & 2.08 & 1.80 & 0.82 & 1.24 & 1.65 & 2.17 & 17.36 & 0.93 \\
$\Lambda$ Importance Weighted $(\mu=20)$ & 4.08 & 12.76 & 0.83 & 1.24 & 1.66 & 2.19 & 173.11 & 0.93 \\
\hline
\end{tabular}

Notes: Estimates for $\Lambda$ are based on $=$ utility normalization.

We remind readers that our preferred specification is the asymmetric CRRA, and that we present the results for both CRRA and CARA forms in Table 4 for illustrative purposes. Comparing across utility forms, we see that the loss aversion measures tend to be greater for the CARA compared to CRRA. These results are consistent with the fact that greater curvature for the CARA utility function requires much larger estimates of $\lambda$ to achieve similar certainty equivalents, even with symmetry imposed. The invariance of the CARA estimates of loss aversion with respect to scale (in the sense that they are greater than unity) and the corresponding lack of that invariance of the CRRA is worth noting. Equally, however, the CARA estimates are not intrinsically more stable, they are just consistently greater than unity. In contrast, the CRRA specification has progressively increasing values of $\Lambda$ as higher absolute payoffs are given greater weight. Under our preferred weighting $(\mu=10)$ around $71 \%$ of respondents have $\Lambda>1$. Thus, our importance weighting estimates suggest that the majority of respondents are loss averse (by the criteria $\Lambda>1$ ). Yet, for $\mu=5$, there appear to be a majority of individuals that are moderately "non-loss averse". Most studies do not report the proportions of those above and below unity, but we would conclude that the CRRA results in lower estimates of loss aversion compared to many other studies. This is consistent with the tendency of loss aversion measures to be volatile (Wakker, 2010 Section 9.5).

The results above illustrate that, for CRRA, conclusions about loss aversion are not invariant to choices of $\mu$. Thus, researchers' judgements about the relative importance of payoffs continue to be needed when deciding on whether people are generally loss averse. Should we conclude that CARA 
is a preferred form when evaluating loss aversion? Our own view is no. While we recognise the desirability of removing all ambiguity about loss aversion, such a conclusion would be driven by a desire to see black and white where there is mostly grey. Researchers' preoccupation with testing $\lambda=1$ vs $\lambda>1$ may have obscured the fact that the measurement of loss aversion is of interest even if data does not unambiguously support overwhelming loss aversion at all scales. We believe researchers should entertain the idea that loss aversion may only be a phenomena at higher scales.

Our CRRA model is strongly preferred to the CARA form and we would not be predisposed towards using a model that is less supported by the data purely because it delivers unambiguous findings with regard to loss aversion. The CRRA results above show that for $\mu=5$, there is a weak tendency towards being "non-loss averse" whereas for $\mu>10$ there is predominantly mild loss aversion. This illustrates that under reasonable weightings of payoffs that the loss aversion exhibited by our respondents is lower than reported in many studies (e.g. 2.8 reported in Tversky and Kahneman, 1992). Nonetheless, for practical purposes there remains a tendency towards loss aversion at scales "that matter". That said, we have only explored two cannoical value forms, and that the investigation of other forms may yeild results that give loss aversion at all scales while being fully supported by the data.

\subsection{Discussion and Practical Implementation}

The method that we have introduced allows researchers to draw conclusions about the overall nature of loss aversion while recognising that in general the scale of losses and gains matter. The previous literature has recognised that there is scale dependency when assessing loss aversion (Abdellaoui et al., 2007). However, the fact that this point has been made before does not mean that it has been fully absorbed or understood. Therefore, at the risk of reiterating a point that has already been appreciated by many, researchers should not start with an aribitrary basis and focus on whether $\lambda$ exceeds unity as a reliable indicator of loss aversion unless they are willing to impose restrictions that may not be supported by the data. Since scale in general matters, we have proposed a straight forward approach which is to aggregate over scales to arrive at a global measure of loss aversion. In our empirical study, we demonstrate and provide practical guidance on how to undertake calculations to that purpose. Importantly, our approach requires no consideration of scale prior to estimation, and can be employed after estimation. Thus, researchers can proceed to estimate their models using any basis utility and by ignoring exchange rates or denomination (e.g. pounds or pence). However, when considering the degree of loss aversion (or otherwise) researchers need to decide on a weighting scheme that broadly reflects the scale at which decision makers generally work. Having decided on these values (or even a range of values) they can proceed to make assessments about the degree of loss 
aversion. In our view this approach leads to far more nuanced but informative examination of loss aversion that simply claiming that loss aversion is equal to specific value.

\section{Remarks and Conclusions}

This paper has principally addressed the identification of loss aversion (the pivot parameter) within PT. We have discussed the point dependent nature of identification of loss aversion and have argued that researchers should not automatically impose symmetry just because of the apparent simplicity it affords. If symmetry is not supported statistically by data then we believe it should not be imposed, and researchers should be aware and explicit about their normalization restrictions. They should also be cognizant of the requirement that normalizations require real and not just nominal equality in order to be comparable from one study to another. The applied literature has so far given little acknowledgment of this point, while reporting values of parameters that are taken to support 'loss aversion'. As an alternative to the selection of a single normalization point, this paper has proposed that averaging over a weighted set of values as a sensible alternative, and accordingly developed an approach based on Gamma importance weightings.

Our approach was applied within a PT framework to a sample farmers using an HBL, with the results supporting a large and growing body of literature that suggests that modelling choices under risks requires a non-linear probability transformations approach consistent with PT. It also generally supported concave value functions in the gain domain and convex value functions in the loss domain, along with a previously observed tendency for the loss domain to have less curvature than the gain domain. However, while there is a general tendency to support the 'stronger' aspects of PT such as inverse-S probability weightings, there is also considerable heterogeneity. This underlines the growing recognition that researchers should allow for substantial respondent heterogeneity when modelling risk, rather than a priori imposing average conditions on everybody.

Within this paper, the CRRA value form was preferred to the CARA, which in turn rejected the symmetry restriction employed in many studies. However, the CRRA form did not favour downward pivoting of the value function for most respondents with symmetry imposed, nor under averaging, where high importance weights were given to low absolute payoffs. However, for importance weights giving reasonable mass to higher values, these suggested downward normalized rotations consistent with what some studies have identified as 'loss aversion'. By contrast, the less favoured CARA form indicated greater downward pivoting of utility in the loss domain. This findings serves as a reminder that pivot parameter estimates depend both on the utility curvature and functional form used to model that curvature, an observation that remains true even under symmetry.

Finally, although the CRRA value form is our preferred specification for this data set, we acknowl- 
edge that there are legitimate questions regarding its general suitability. Functional forms, such as the modified version of the power family (see Wakker, 2010, for details) might perform better. However, our central conclusions do not depend on choices of functional form. The ultimate conclusions about the degree of loss aversion may change if we examine more general functional forms, yet the size dependence issues highlighted in the paper remain. Here we do not investigate additional parametric functional forms because our focus is not on model selection. There is, however, obvious scope to extend the analysis presented in this paper to a wider set of functional forms. We leave this challenge for the interested reader to consider. 


\section{Notes}

${ }^{1}$ For simplicity we adopt these two descriptors throughout. However, as discussed in Wakker (2008), the CRRA specification used here is restricted to the 'power forms' where the power parameters are strictly positive since negative powers cannot apply where utility has a negative domain, as a monotonically increasing utility function over the real numbers cannot be constructed for any power function more concave than the log (the limiting case of the CRRA)

${ }^{2}$ For example, take basis utility defined by the piecewise functions

$$
u_{1}^{-}(z)=\left(1-e^{s z}\right) 2 z+e^{s z} z ; u_{1}^{+}(z)=z ; \text { and } \lambda_{1}=1
$$

where $s$ is some large number. $u_{1}^{-}(z)$ is negative, and has a derivative that is positive for all $z<0$ and converges on one at zero . Next define

$$
u_{2}^{-}(z)=z ; u_{2}^{+}(z)=z \text { and } \lambda_{2}=2
$$

Under the Köbberling and Wakker (2005) normalisation, $\tilde{\lambda}_{1}=1$ for the first utility function and $\tilde{\lambda}_{2}=2$ for the second. But, in practical terms the utility functions are almost identical

${ }^{3}$ We note that $u^{-\prime}(0)$ and $u^{+\prime}(0)$ are not required to exist for the piecewise utility function in equation (1). It may be that:

a1 $\lim _{x \rightarrow 0} u^{-\prime}(-z)$ or $\lim _{x \rightarrow 0} u^{+\prime}(z)$ do not exist and neither does $\lim _{x \rightarrow 0} \gamma_{U}(z)$

a2 $\lim _{x \rightarrow 0} u^{-\prime}(-z)$ and $\lim _{x \rightarrow 0} u^{+\prime}(z)$ do not exist but $\lim _{x \rightarrow 0} \gamma_{U}(z)$ still exists (denoted $\vec{\gamma}_{U}(0)$ )

a3 $\lim _{x \rightarrow 0} u^{-\prime}(-z)=l>0$ and $\lim _{x \rightarrow 0} u^{+\prime}(z)=u>0$ for finite $u$ and $l$ and define $\vec{\gamma}_{U}(0)=\frac{l}{u}$

a4 Positive finite derivatives exist at 0 , subject to $u^{-\prime}(0)=l>0$ and $u^{+\prime}(0)=u>0$ where $\gamma_{U}(0)$ $=\vec{\gamma}_{U}(0)=\frac{l}{u}$.

${ }^{4}$ A detailed explanation of this point is provided in Appendix D.

${ }^{5}$ Note, only under very special conditions is loss aversion, identifiable and therefore a testable component of the model as we specify it.

${ }^{6} \mathrm{We}$ also ran models with wider intervals for the probability weighting functions, which lead to slightly more extreme probability weightings for a small number of individuals, and slightly more extreme values for the utility function parameters. However, the findings that we present were not altered in any substantive sense.

${ }^{7}$ For details see http://www.mywage.org/ghana/home/salary/minimum-wages 
${ }^{8}$ Our use of a binding round with which to ensure incentive compatability is standard within the literature. However, there is a significant degree of variation in how incentive compatability is achieved. For example, several authors ensure that the participation fee is the same magnitude as the maximum loss that can be realised during an experiment (e.g., Harrison and Rutström, 2009; Andersen et al., 2010;Liu and Huang, 2013). Some experiments do not enforce the payment for a loss if this is the binding prospect that is played (e.g., Ward and Singh, 2015). Finally, there are also examples of completely hypothetical prospects with no effort to enforce incentive compatability (e.g., Booij et al., 2010).

${ }^{9}$ We note that there are always aspects of experimental design and implementation that can be improved and the experiment presented in this paper is no different. For this reason, we positively advocate that researchers try the method presented in this paper with existing or new data sets.

${ }^{10}$ The issue of what is considered "large" prompts consideration of the fact that the degree of loss aversion may be a function of income or weath, even though the reference point of zero remains for prospects. We do not explore this issue here. However, we note that were we simply to take the value functions here, we would redefine large using larger values of $\mu$ which would translate to much larger estimates of loss aversion (though we would not contend that this should be done) 


\section{References}

Abdellaoui, M., Bleichrodt, H. and Paraschiv, C. (2007). Loss Aversion Under Prospect Theory: A Parameter-Free Measurement. Management Science, 53(10): 1659-1674.

Andersen, S., Harrison, G.W., Lau, M.I. and Rutström, E.E. (2010). Behavioral Econometrics for Psychologists. Journal of Economic Psychology, 31: 553-576.

Balcombe, K.G. and Fraser, I.M. (2015). Parametric preference functionals under risk in the gain domain: A Bayesian analysis. Journal of Risk and Uncertainty, 50: 161-187.

Booij, A. S., van Praag, M.S. and van de Kuilen, G. (2010). A Parametric Analysis of Prospect Theory's Functionals for the General Population. Theory and Decision, 68: 115-148.

Chateauneuf, A. and Wakker, P.P. (1999). An axiomatization of cumulative prospect theory for decision under risk. Journal of Risk Uncertainty, 18:137-145.

Ert, E. and Erev, I. (2013). On the Descriptive Value of Loss Aversion in Decisions Under Risk: Six Clarifications. Judgement and Decision Making, 8(3): 214-235.

Harrison, G.W. and Rutström, E. (2009). Expected utility and prospect theory: one wedding and a decent funeral. Experimental Economics, 12(2), 133-158.

Harrison, G.W., Humphrey, S. and Verschoor, A. (2010). Choice Under Uncertainty: Evidence From Ethiopia, India And Uganda. Economic Journal, 120(543): 80-104.

Harrison, G.W. and Swarthout, J.T. (2016). Cumulative Prospect Theory in the Laboratory: A Reconsideration. Memo.

Köbberling, V. and Wakker, P.P. (2005). An index of loss aversion. Journal of Economic Theory, $122(1): 119-131$.

Liu, E.M. and Huang, J. (2013). Risk Preferences and Pesticide Use by Cotton Farmers in China. Journal of Development Economics, 103: 202-215.

Nilsson H., Rieskampa J. and Wagenmakers E.J. (2011). Hierarchical Bayesian parameter estimation for cumulative prospect theory. Journal of Mathematical Psychology, 55: 84-93.

Scheibehenne, B. and Pachur, T. (2015). Using Bayesian Hierarchical Parameter Estimation to Assess the Generalizability of Cognitive Models of Choice. Psychonomic Bulletin and Review, 22: $391-4-7$. 
Schmidt, U. and Traub, S. (2002). An Experimental Test of Loss Aversion. Journal of Risk and Uncertainty, 25: 233-249.

Stott, H.P. (2006). Cumulative prospect theory's functional menagerie. Journal of Risk and Uncertainty, 32: 101-130.

Tanaka, T., Camerer, C.F. and Nguyen, Q. (2010). Risk And Time Preferences: Linking Experimental And Household Survey Data From Vietnam. American Economic Review, 100(1): $557-571$.

Train K. D. (2009) Discrete Choice Methods with Simulation. Second Edition, Cambridge University Press.

Tversky, A. and Kahneman, D. (1992). Advances in Prospect Theory: Cumulative Representation of Uncertainty. Journal of Risk and Uncertainty, 5: 297-323.

Wakker P.P. (2008) Explaining the characteristics of the Power (CRRA) Utility Family. Health Economics, 17: 1329-1344

Wakker, P.P. (2010). Prospect Theory: For Risk and Ambiguity, Cambridge University Press.

Wakker, P.P. and Tversky, A. (1993). An axiomatization of cumulative prospect theory. Journal of Risk and Uncertainty,7(2): 147-175

Walasek, L. and Stewart, N. (2015). How to Make Loss Aversion Disappear and Reverse: Tests of the Decision by Sampling Origin of Loss Aversion. Journal of Experimental Psychology: General, 144(1): 7-11.

Ward, P.S., and Singh, V. (2015). Using Field Experiments to Elicit Risk and Ambiguity Preferences: Behavioural Factots and the Adoption of New Technologies in Rural India, Journal of Development Studies, 51(6): 707-724.

Zank, H. (2010). On Probabilities and Loss Aversion. Theory and Decision, 68: 243-261. 
Figure 1: Representation of the Prospects Used

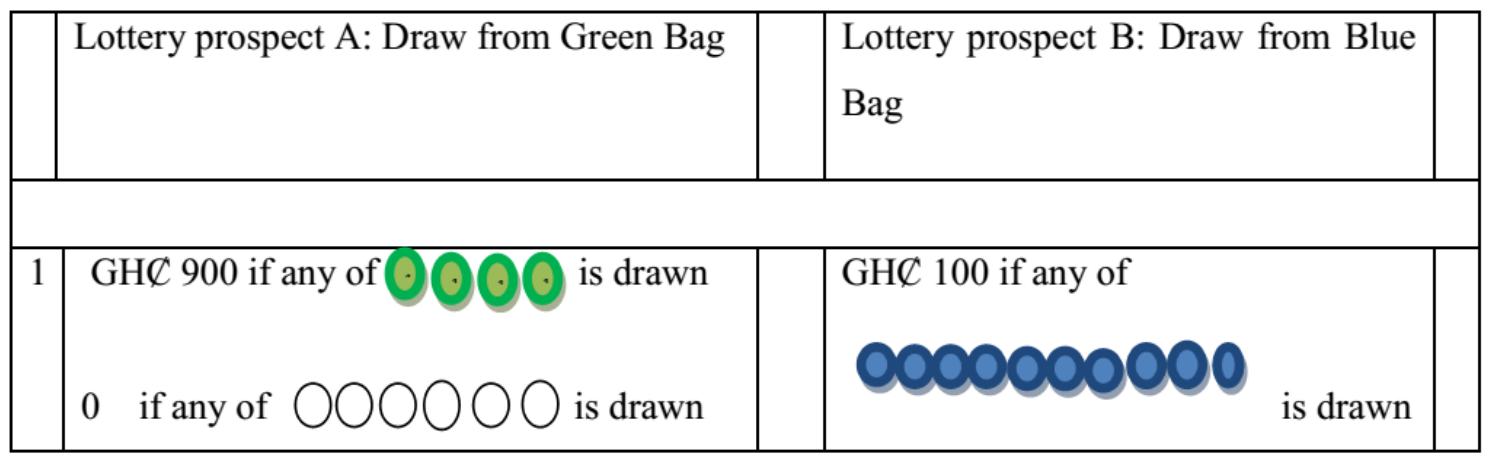

Figure 2: Probability Weightings

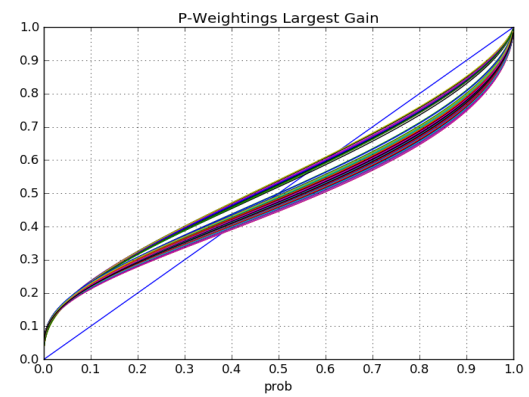

\section{CRRA Functional}

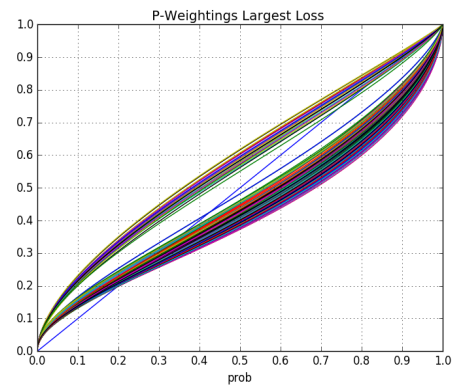


Figure 3: Importance Weighting Functions

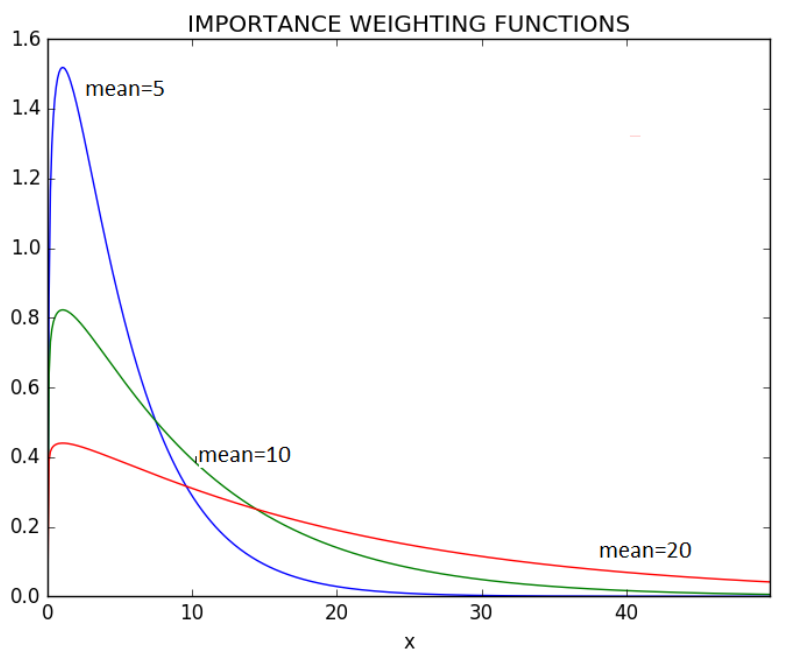




\section{For Online Publication}

\section{Appendix A:}

Table A1: Sample Descriptive Statistics

\begin{tabular}{lllll}
\hline Variables & \multicolumn{5}{c}{ Ranges } \\
\hline Gender & Male & Female & & \\
Freq (\%) & $255(66.4)$ & $129(33.6)$ & & \\
Age (Years) & $30-40$ & $41-50$ & $51-60$ & $>60$ \\
Freq (\%) & $78(20.3)$ & $157(40.8)$ & $118(30.7)$ & $31(8.2)$ \\
Education & None & Primary & Secondary & Higher \\
Freq (\%) & $53(13.8)$ & $89(23.2)$ & $167(43.5)$ & $75(19.5)$ \\
Marital Status & Single & Married & Divorced & Other \\
Freq (\%) & $17(4.4)$ & $324(84.4)$ & $13(3.4)$ & $30(7.8)$ \\
Family Size & $1-5$ & $6-10$ & $11-15$ & \\
Freq (\%) & $150(39.3)$ & $203(53)$ & $29(7.3)$ & \\
Crop (Type) & Maize & Cassava & Plantain & Vegetables \\
Freq (\%) & $280(72.9)$ & $322(83.9)$ & $230(59.9)$ & $131(34.1)$ \\
Faming (Years) & $20-30$ & $31-40$ & $41-50$ & \\
Freq (\%) & $319(83.1)$ & $63(16.4)$ & $2(0.5)$ & \\
Sources Finance & Informal Credit & Formal Credit & Savings & Other \\
Freq (\%) & $55(14.3)$ & $51(13.3)$ & $273(71.1)$ & $5(1.3)$ \\
Extension Contact & Very Often & Often & Occasional & None \\
Freq (\%) & $122(31.8)$ & $145(37.8)$ & $102(26.6)$ & $15(3.9)$ \\
\hline
\end{tabular}




\section{Appendix B:}

Table B1: Gain Domain

Lottery A (Green Bag) Lottery B (Blue Bag)

\begin{tabular}{lllllllll} 
Task & $\mathbf{z}$ & $\mathbf{p}$ & $\mathbf{z}$ & $\mathbf{p}$ & $\mathbf{z}$ & $\mathbf{p}$ & $\mathbf{z}$ & $\mathbf{p}$ \\
\hline 1 & 200 & 0.9 & 100 & 0.1 & 900 & 0.1 & 0 & 0.9 \\
2 & 800 & 0.4 & 0 & 0.6 & 300 & 1.0 & 0 & 0 \\
3 & 700 & 0.6 & 0 & 0.4 & 500 & 0.8 & 100 & 0.2 \\
4 & 700 & 0.2 & 200 & 0.8 & 800 & 0.4 & 0 & 0.6 \\
5 & 600 & 0.2 & 0 & 0.8 & 200 & 1 & 0 & 0 \\
6 & 300 & 0.8 & 0 & 0.2 & 0 & 0 & 200 & 1 \\
7 & 0 & 0 & 200 & 1 & 300 & 0.8 & 0 & 0.2 \\
8 & 500 & 1 & 0 & 0 & 1000 & 0.5 & 0 & 0.5 \\
9 & 900 & 0.4 & 0 & 0.6 & 100 & 1 & 0 & 0 \\
10 & 400 & 0.2 & 200 & 0.8 & 800 & 0.5 & 0 & 0.5 \\
11 & 1000 & 0.4 & 100 & 0.6 & 800 & 0.1 & 300 & 0.9 \\
12 & 700 & 0.1 & 200 & 0.9 & 1000 & 0.4 & 0 & 0.6 \\
13 & 900 & 0.2 & 0 & 0.8 & 200 & 1 & 0 & 0 \\
14 & 800 & 0.2 & 200 & 0.8 & 900 & 0.5 & 0 & 0.5 \\
15 & 200 & 0.6 & 100 & 0.4 & 900 & 0.1 & 0 & 0.9 \\
16 & 900 & 0.6 & 200 & 0.4 & 700 & 0.5 & 400 & 0.5 \\
\hline & & & & & & &
\end{tabular}


Table B2: Loss Domain

\begin{tabular}{|c|c|c|c|c|c|c|c|c|}
\hline \multirow[b]{2}{*}{ Task } & \multicolumn{2}{|c|}{ Lottery } & \multicolumn{2}{|c|}{ A (Orange Bag) } & \multicolumn{4}{|c|}{ Lottery B (Red Bag) } \\
\hline & $\mathbf{z}$ & $\mathbf{p}$ & $\mathbf{z}$ & $\mathrm{p}$ & $\mathbf{z}$ & $\mathrm{p}$ & $\mathbf{z}$ & $\mathbf{p}$ \\
\hline 1 & 300 & 0.8 & 900 & 0.2 & 300 & 0.1 & 500 & 0.9 \\
\hline 2 & 400 & 0.8 & 700 & 0.2 & 0 & 0.3 & 800 & 0.7 \\
\hline 3 & 100 & 0.7 & 800 & 0.3 & 300 & 0.6 & 400 & 0.4 \\
\hline 4 & 100 & 0.3 & 600 & 0.7 & 400 & 1 & 0 & 0 \\
\hline 5 & 400 & 0.6 & 900 & 0.4 & 600 & 1 & 0 & 0 \\
\hline 6 & 100 & 0.7 & 300 & 0.3 & 0 & 0.9 & 800 & 0.1 \\
\hline 7 & 0 & 0 & 400 & 1 & 0 & 0.5 & 800 & 0.5 \\
\hline 8 & 200 & 1 & 0 & 0 & 0 & 0.7 & 500 & 0.3 \\
\hline 9 & 0 & 0.4 & 700 & 0.6 & 100 & 0.9 & 700 & 0.1 \\
\hline 10 & 0 & 0.5 & 500 & 0.5 & 100 & 0.4 & 200 & 0.6 \\
\hline 11 & 0 & 0.3 & 1000 & 0.7 & 300 & 0.7 & 800 & 0.3 \\
\hline 12 & 0 & 0.5 & 800 & 0.5 & 200 & 0.9 & 900 & 0.1 \\
\hline 13 & 0 & 0.8 & 900 & 0.2 & 0 & 0.2 & 300 & 0.8 \\
\hline 14 & 200 & 0.3 & 900 & 0.7 & 0 & 0 & 600 & 1 \\
\hline 15 & 0 & 0 & 400 & 1 & 0 & 0.4 & 800 & 0.6 \\
\hline 16 & 200 & 0.9 & 800 & 0.1 & 0 & 0 & 300 & 1 \\
\hline
\end{tabular}

Note: All payoffs (z) are negative values 
Table B3: Mixed Domain

Lottery A (Green Bag) Lottery B (Blue Bag)

\begin{tabular}{lllllllll} 
Task & $\mathbf{z}$ & $\mathbf{p}$ & $\mathbf{z}$ & $\mathbf{p}$ & $\mathbf{z}$ & $\mathbf{p}$ & $\mathbf{z}$ & $\mathbf{p}$ \\
\hline 1 & 300 & 0.8 & -700 & 0.2 & 900 & 0.2 & -400 & 0.8 \\
2 & 300 & 0.5 & -100 & 0.5 & 200 & 0.3 & 0 & 0.7 \\
3 & 0 & 0 & -400 & 1 & 300 & 0.4 & -900 & 0.6 \\
4 & 0 & 0.9 & -900 & 0.1 & 900 & 0.3 & -700 & 0.7 \\
5 & 300 & 0.2 & -900 & 0.8 & 400 & 0.5 & -500 & 0.5 \\
6 & 100 & 1 & 0 & 0 & 100 & 0.5 & -700 & 0.5 \\
7 & 100 & 1 & 0 & 0 & 1000 & 0.3 & -400 & 0.7 \\
8 & 700 & 0.6 & -800 & 0.4 & 900 & 0.5 & -800 & 0.5 \\
9 & 900 & 0.3 & -200 & 0.7 & 0 & 0 & -200 & 1 \\
10 & 100 & 1 & 0 & 0 & 800 & 0.3 & -100 & 0.7 \\
11 & 100 & 1 & 0 & 0 & 600 & 0.6 & -600 & 0.4 \\
12 & 200 & 0.2 & -500 & 0.8 & 200 & 0.3 & -900 & 0.7 \\
13 & 500 & 0.6 & -100 & 0.4 & 900 & 0.5 & -200 & 0.5 \\
14 & 0 & 0 & -100 & 1 & 700 & 0.2 & -100 & 0.8 \\
15 & 600 & 0.8 & -700 & 0.2 & 900 & 0.1 & -1000 & 0.9 \\
16 & 0 & 0 & -200 & 1 & 600 & 0.5 & -900 & 0.5 \\
\hline
\end{tabular}




\section{Appendix C:}

Observing the second order Taylor expansion

$$
\ell(z) \simeq\left(\ell(\mu)-\ell^{\prime}(\mu) \mu+\frac{\ell^{\prime \prime}(\mu)}{2} \mu^{2}\right)+\left(\ell^{\prime}(\mu)-\ell^{\prime \prime}(\mu)(\mu) \mu\right) z+\frac{\ell^{\prime \prime}(\mu)}{2} z^{2}
$$

and letting $g^{*}(\mu)=\frac{\alpha}{\beta} \frac{1-e^{-\beta \mu}}{1-e^{-\alpha \mu}}$ and $g(\mu)=\frac{1-e^{-\beta \mu}}{1-e^{-\alpha \mu}}$, if we define for the $\log$ of $\ell(\mu)=\ln g^{*}(\mu)$ we then have $\ell_{1}(\mu)=\ell^{\prime}(\mu)=\frac{g^{\prime}(\mu)}{g(\mu)}$ and $\ell_{2}(\mu)=\ell^{\prime \prime}(\mu)=\frac{g^{\prime \prime}(\mu)}{g(\mu)}-\ell_{1}(\mu)^{2}$, such that the approximation in the text is:

$$
\begin{aligned}
& \ell(z)=\underbrace{\left(\ell(\mu)-\ell_{1}(\mu) \mu+\frac{\ell_{2}(\mu)}{2} \mu^{2}\right)}_{c_{0}}+\underbrace{\left(\ell_{1}(\mu)-\ell_{2}(\mu) \mu\right)}_{c_{1}} z+\underbrace{\frac{\ell_{2}(\mu)}{2} z^{2}}_{c_{2}} \\
& g^{\prime}(\mu)=\frac{\left[\beta e^{-\beta \mu}-\alpha g(\mu) e^{-\alpha \mu}\right]}{1-e^{-\alpha \mu}} \\
& \ell_{1}(\mu)=\frac{\left[\beta e^{-\beta \mu}-\alpha g(\mu) e^{-\alpha \mu}\right]}{1-e^{-\beta \mu}} \\
& \ell_{2}(\mu)=\frac{-\beta^{2} e^{-\beta \mu}+\alpha^{2} g(\mu) e^{-\alpha \mu}-2 \alpha g^{\prime}(\mu) e^{-\alpha \mu}}{1-e^{-\beta \mu}}-\ell_{1}(\mu)^{2}
\end{aligned}
$$




\section{Appendix D: Preserving Denomination Independence}

If we denominate a utility function in two currencies $\mathbf{P}$ and $p$, where $z \mathbf{P} \equiv \varphi z p$ then if we require that (if they reflect the same real ordering) $U_{\mathbf{P}}(z) \equiv U_{p}(\varphi z)$. A utility function $U(\varphi, z)$ that preserves ordering in real terms across different denominations requires that $U(\varphi, \varphi z) \equiv U(1, z)$ for any $\varphi>0$ and all $z$. If a denomination dependent normalized coefficient $\tilde{\lambda}(\varphi, z)$ is invariant to the real point of normalization rather than the nominal point it requires that $\tilde{\lambda}(\varphi, \varphi z)=\tilde{\lambda}(1, z)$. Therefore, we should not expect in general that $\tilde{\lambda}(\varphi, z)=\tilde{\lambda}(1, z)$. The importance of denomination dependence is discussed by Wakker (2010) and Harrison and Swarthout (2016) albeit it is referred to as the exchange rate assumption.

For the CRRA form, we see that for $\delta=1$ where $z \geq 0$ and 0 otherwise, we can specify:

$$
U(\varphi, z)=\delta z^{\alpha}-(1-\delta) \lambda \varphi^{\alpha-\beta}(-z)^{\beta}
$$

The normalized value at point $z>0, \tilde{\lambda}^{\tau}(\varphi, z)=\varphi^{\alpha-\beta} \lambda \frac{z^{\beta}}{z^{\alpha}} \Rightarrow \tilde{\lambda}^{\tau}(\varphi, \varphi z)=\lambda \frac{z^{\beta}}{z^{\alpha}}$ which independent of $\varphi$.

For the CARA form, the value of $\lambda$ for some basis utility will be invariant to denomination, since we can write it in a denomination dependent form as

$$
U(\varphi, z)=\left(\delta \frac{\varphi\left(1-e^{-\frac{\alpha}{\varphi} z}\right)}{\alpha}-(1-\delta) \lambda \frac{\varphi\left(1-e^{\frac{\beta}{\varphi} z}\right)}{\beta}\right)
$$

then $\tilde{\lambda}^{\tau}(\varphi, z)=\lambda \frac{\alpha}{\beta} \frac{\left(1-e^{-\frac{\beta}{\varphi} z}\right)}{\left(1-e^{-\frac{a}{\varphi} z}\right)} ; \tilde{\lambda}^{\tau}(\varphi, \varphi z)=\lambda z_{z^{\alpha}}$ independent of $s$.

These results simply serve to remind us that if normalized $\tilde{\lambda}$ are calculated, they need to be normalized in accordance with the scaling of the data, so that it is done in real and not nominal terms. Finally, since $h_{n}(\varphi \mu, \varphi m)=\frac{\Gamma\left(\frac{\mu}{\mu-m}+n\right)(\varphi \mu-\varphi m)^{n}}{\Gamma\left(\frac{\mu}{\mu-m}\right)}=\varphi^{n} h_{n}(\mu, m)$ any integral approximation requires scaling of the importance function (in terms of both the mean and mode) to preserve real comparisons. We note that by denomination dependence readers should be clear that we do not just mean the denomination as used for elicitation. If two researchers with exactly the same data use different denominations when estimating the models (e.g. one runs the models using pounds and another with pence) they need to be clear about this denomination in order to have comparative results. 


\section{Appendix E: Parameter Intepretation}

Table 1E: Parameter Interpretations

$\alpha$

CRRA : $\uparrow$ increases $:$ risk seeking for gains, $<1=$ concave, $1=$ linear, $>1=$ convex

CARA : $\uparrow$ decreases : risk seeking for losses, $>0=$ concave, $1=$ linear, $<0=$ convex

CRRA : $\uparrow$ increases $:$ risk aversion for gains, $<1=$ convex, 1 linear, $>1=$ concave

CARA : $\uparrow$ decreases $:$ risk aversion for losses, $>0=$ convex, 1 linear, $<0=$ concave

$\gamma \quad \uparrow$ increases $: \mathrm{S}$ behavior of weighting for largest gains, $<1=\mathrm{IS}, 1=$ linear, $>1=\mathrm{S}$

$\delta \quad \uparrow$ decreases $:$ weighting of largest gains, $<1=$ optimism, $1=$ linear, $>1=$ pessimism

$\varepsilon \quad \uparrow$ increases $: \mathrm{S}$ behavior of weighting for largest losses, $<1=\mathrm{IS}, 1=$ linear, $>1=\mathrm{S}$

$\eta \quad \uparrow$ decreases : weighting of largest losses, $<1=$ pessimism, $1=$ linear, $>1=$ optimism

$\lambda \quad \uparrow$ increases : weighting of loss to gain for value functions

$\phi \quad \uparrow$ increases : probability of choice given preference under PT (signal/noise ratio)

Note: IS overweights small probabilities/overweights large probabilities (S does opposite)

Increase weightings largest gains (losses)/decrease weightings smallest gain (losses) etc. 


\section{Appendix F: Convergence and Posterior Prediction}

Convergence is checked visually, but also automatically by conducting modified t-tests subject to Bonferroni corrections, for the mean parameters of the first thousand and last thousand using a $5 \%$ level of significance. Similarly we look at the Gelman and Rubin Rhat statistics for each chain using the first 1000 and last 1000 draws. For our preferred model we also ran two separate chains and checked the Gelman and Rubin Rhat statistic which had a maximum of 1.04 for one of the parameters (anything below 1.10 being considered reasonable). Figure F1, gives the Trace Plot for the latent means for the preferred model, which is typical of the Traceplots for each of the models. This type of traceplot is consistent with good mixing, and convergent results.

Figure F1: Typical Trace Plot

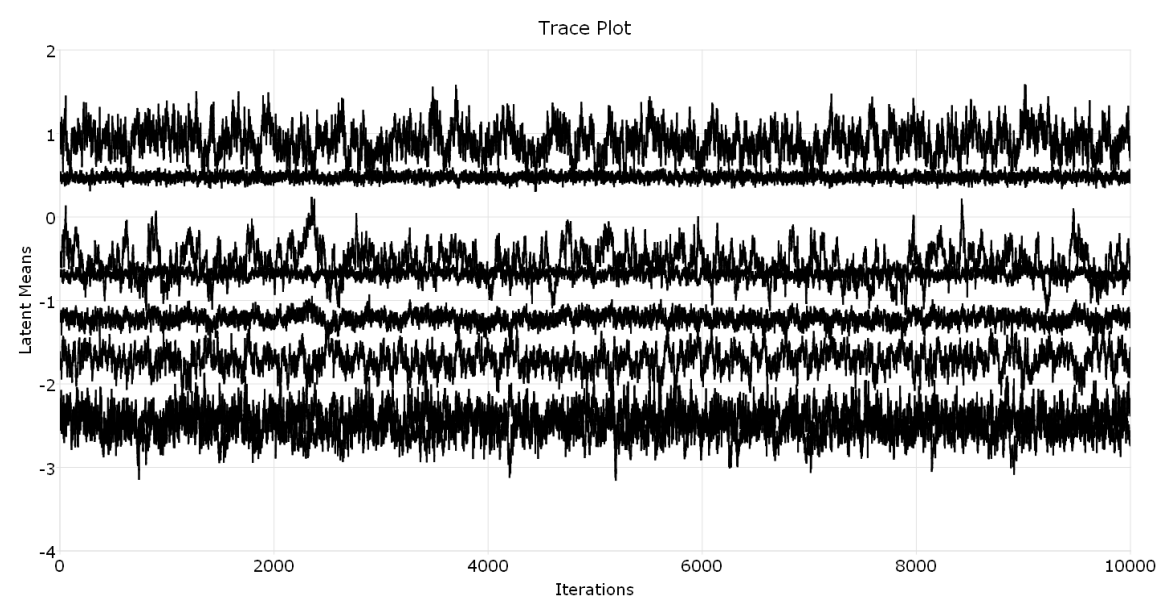

The overall predictive accuracy over all choices by all individuals was $78 \%$. The predictive accuracy with respect to individuals is illustrated Figure F2 below. On the horizontal axis the predictive accuracy is given with the line showing the proportion of respondents with a better rate of prediction than the associated coordinate on the horizontal axis. This shows that 100 percent (proportion of 1) individuals had better than $50 \%$ (the rate of prediction for random choices). Thus, this model does better than random prediction for all individuals in the sample. Naturally, the proportion falls with predictve accuracy. Thus, around $75 \%$ of individuals had there choices correctly predicted above $60 \%$ of the time, but with only $25 \%$ of individuals having there choice predicted in more than $70 \%$ of choices. 
Figure F2: Posterior Predictive Accuracy

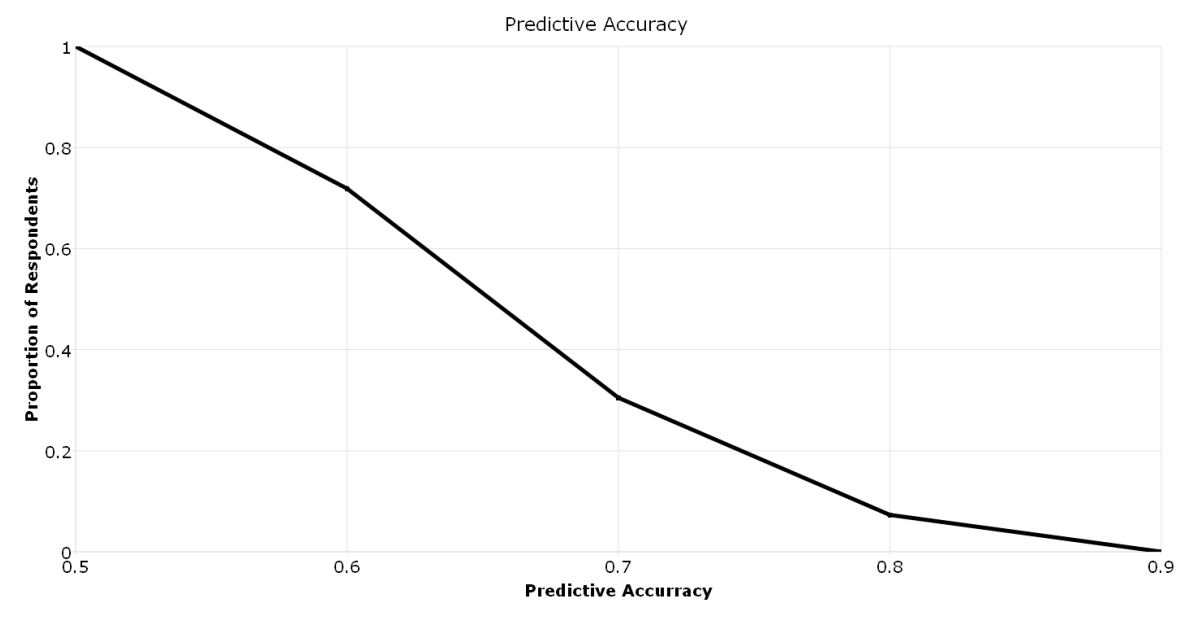

